\title{
Towards an Improvement of the SABIAN Humanoid Robot: from Design to Optimization
}

\author{
G. G. Muscolo ${ }^{1,2, *}$, C. T. Recchiuto ${ }^{1,3}$, K. Hashimoto ${ }^{4}$, P. Dario ${ }^{2}$, A. Takanishi ${ }^{4,5}$ \\ ${ }^{1}$ Humanot s.r.1., Via Armando Spadini, 16, 50142, Firenze (FI), Italy \\ ${ }^{2}$ The Biorobotics Institute, Scuola Superiore Sant'Anna, Viale Rinaldo Piaggio, 34, 56025, Pontedera (PI), Italy \\ ${ }^{3}$ Department of Mechanical Engineering, University of Roma Tor Vergata, Via Del Politecnico, 1, 00133, Roma (RM) \\ ${ }^{4}$ Department of Modern Mechanical Engineering, Waseda University, 17 Kikui-cho, Shinjuku-ku, Tokyo 162-0044, Japan \\ ${ }^{5}$ Humanoid Robotics Institute, Waseda University, 2-2 Wakamatsu-cho, Shinjuku-ku, Tokyo 162-8480, Japan
}

\begin{abstract}
In this paper the humanoid robot SABIAN (Sant'Anna BIpedal humANoid) is presented and analyzed in details. In particular, the differences between the SABIAN platform and the WABIAN-2 (WAseda BIpedal humANoid), its original Japanese version, are underlined. Some innovative solutions, as the exact calculation of the error mass and the adoption of a new shaft with incorporated joint used to transfer motion, allowed to improve the performances of the robot. Other works, such as the development of a harvesting system for recovering energy, are planned for the next future in order to optimize the platform.
\end{abstract}

Keywords Biped Robots, Locomotion, Humanoids, WABIAN-2, SABIAN, ZMP

\section{Introduction}

Humanoid robotics is one of the most interesting and most active fields in the whole engineering sector, since its multidisciplinary and its prospective development in a next future. Despite all the efforts, the development of a humanoid robot able to operate in a real environment is an open challenge for the robotic research.

In the last years, the research was mostly focused on the cooperation and on the communication, between humanoids and humans[1].Other kinds of approaches concern the simulation and the reproduction of human movements such as walking motions[2] or dance moves[3].

The biped locomotion is one of the most complex fields of humanoid robotics. Since 1960, many different research gro ups all over the world started to investigate the stabilization during a walking motion. Prof. Vukobratović formalized the model of the robotic/human motion by studying the forces acting on the foot[4] and introducing the concept of the Zero Moment Point (ZMP). Most of the researches related to locomotion were based on this concept, trying to define the equation of motion, by using the concept of the inverse pendulum[5] or using the Fourier Transformer to compensate the moment around the ZMP with the torso orientation[6].

* Corresponding author:

gmuscleg@hotmail.com (G. G. Muscolo)

Published online at http://journal.sapub.org/jmea

Copyright (C) 2012 Scientific \& Academic Publishing. All Rights Reserved
The first humanoid robots were developed as research prototypes by the Waseda University of Tokyo, Japan, in 1973 with the WABOT-project. Since then, WASEDA developed many anthropomorphic robots, as WABOT-2, a robot able to play piano, developed in 1984 and WABIAN (WAseda BIpedal humANoid), a biped robot for the research in the locomotion field[7]. In Europe, the research related to humanoid robots is in a fast expansion, as demonstrated by the German ARMAR III[8], a robot with head, torso and hands mounted on a motorized platform and the EU founded RobotCub[9] research project that allowed the design and development of a humanoid robot with characteristics similar to a child.

Many of these studies were synthesized in the realization of the humanoid platform WABIAN-2[10], and then in its Italian version SABIAN (Sant'Anna BIpedal humANoid). A detailed description of the SABIAN characteristics, in particular compared to WABIAN-2, is presented in this paper. The SABIAN robot moved its first steps in 2010 thanks to the optimization of the center of mass position presented in[11] and to innovative hardware solutions implemented on the SABIAN and presented in this paper. Future steps are oriented to implement some of these solutions on the WABIAN-2 and on the humanoid robots that will be developed in the future.

\section{SABIAN Humanoid Robot: Past and Present}

The first version of WABIAN was developed in 1995, in 
the laboratory of the Waseda University of Tokyo directed by prof. Atsuo Takanishi[7] (Figure 1a). The current version of WABIAN-2 has a height of $1480 \mathrm{~mm}$ and a weight of 63.8 $\mathrm{kg}$ (table 1).

Table 1. Differences between WABIAN-2 \& SABIAN

\begin{tabular}{|c|c|c|}
\hline CHARACTERISTICS & WABIAN-2 & SABIAN \\
\hline Height $[\mathrm{mm}]$ & 1487 & 1300 \\
\hline Weight $[\mathrm{kg}]$ & 64,5 (with batteries) & $\begin{array}{c}40,75 \text { (without } \\
\text { batteries) }\end{array}$ \\
\hline DOF & $\begin{array}{c}\text { Leg 6x2 / Foot 1x2 } \\
\text { (passive) / Waist } 2 \text { / } \\
\text { Trunk 2 / Arm 7x2 / } \\
\text { Hand 3x2 / Neck } 3 \text {. } \\
\text { Total: } 41 . \\
\end{array}$ & $\begin{array}{c}\text { Leg } 7 \times 2 \text { / Foot } 0 / \\
\text { Waist } 2 \text { / Trunk } 2 \\
\text { (not working) / } \\
\text { Arm } 0 \text { / Hand } 0 \text { / } \\
\text { Neck 0. Total: } 16 . \\
\end{array}$ \\
\hline Sensors & $\begin{array}{c}4 \text { Force/Torque } \\
\text { triaxial sensors; } \\
\text { Photo-sensors; } \\
\text { Magnetic incre- } \\
\text { mental Encoders; } \\
\text { Gyro sensors. }\end{array}$ & $\begin{array}{l}2 \text { Force/Torque } \\
\text { triaxial sensors; } \\
\text { Photo-sensors; } \\
\text { Magnetic incre- } \\
\text { mental Encoders. }\end{array}$ \\
\hline Actuators & Servo motors DC. & Servo motors DC. \\
\hline $\begin{array}{c}\text { Motors and Harmonic } \\
\text { drive }\end{array}$ & $\begin{array}{c}\text { Pulley, Belt, Har- } \\
\text { monic drives (HD). } \\
\text { HD Shaft Material: } \\
\text { Aluminum with } \\
\text { high resistance. } \\
\text { Simple shaft design. } \\
\text { Transmission be- } \\
\text { tween shaft and HD } \\
\text { with joint. }\end{array}$ & $\begin{array}{l}\text { Pulley, Belt, } \\
\text { Harmonic drives } \\
\text { (HD). HD Shaft } \\
\text { Material: Steel. } \\
\text { Design of the } \\
\text { shaft with incor- } \\
\text { porated joint. }\end{array}$ \\
\hline Battery & Lithium battery. & Without battery. \\
\hline Feet & $\begin{array}{c}\text { Different versions. } \\
\text { A simple base. Ma- } \\
\text { terial: Plywood. A } \\
\text { complex base with } \\
\text { passive joint[12]. } \\
\text { Material: Alumi- } \\
\text { num. }\end{array}$ & $\begin{array}{c}\text { Unique version. A } \\
\text { simple base. Ma- } \\
\text { terial: Plywood. }\end{array}$ \\
\hline
\end{tabular}

It has a total of 41 degrees, with 6 degrees of freedom for each leg and 2-DoF for the waist that allow a human-like walking motion. While most of the biped robots walk with bent knees, the WASEDA teams, taking into account the importance of the pelvis motion, gait's analysis show that the pelvis motion plays a significant role in human's gait. As the results of these researches, WASEDA team developed a 6-DOF waist-leg system. By using the redundant mechanism, the robot is able to perform a stretch-knee gait as well as human being does. The joint mechanism implemented in WABIAN-2 and SABIAN allows the human-like walking motion.

The computer mounted on the trunk controls the motion of WABIAN-2. It consists of a PCI CPU board and PCI I/O boards. As the I/O boards, HRP interface boards $(16 \mathrm{ch}$ DACs, $16 \mathrm{ch}$ counters, $16 \mathrm{ch}$ PIOs), and 6-axis force/torque sensor receiver board are mounted. The operating system is QNX Neutrino ver. 6.3. The drive system consists of a DC servo motor with an incremental encoder attached to the motor shaft and a photo sensor to detect the basing angle.
Also, each ankle has a 6-axis force/torque sensor, which is used for measuring Ground Reaction Force (GRF) and Zero Moment Point (ZMP).

The realization of the SABIAN (Fig.1b) humanoid robot, started in 2004, was one of the result of the collaboration between Scuola Superiore Sant'Anna and Waseda University of Tokyo, which led to the joint laboratories Robot-AN and Robo-CASA. Since the main purpose of the realization of the SABIAN was the research about locomotion, it was built with a total of 16-DoF, 14 for the legs and 2 for the waist. So, in comparison with the WABIAN-2 joint structure, it has an active joint for the movement of the ankle about the yaw axis while it does not have the passive toe joint actually present in the foot of the WABIAN-2. The sensors and the control architecture are the same of the actual version of the WABIAN-2.

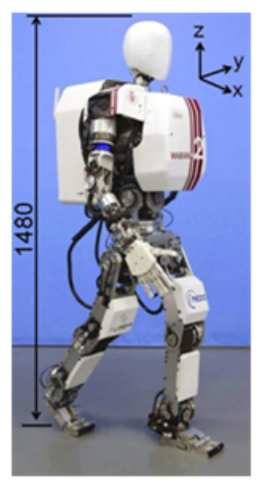

(a)

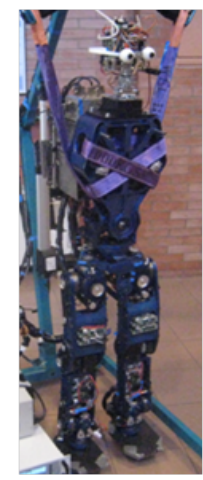

(b)

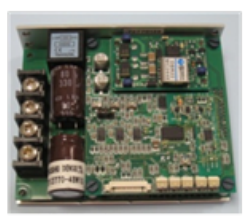

(c)

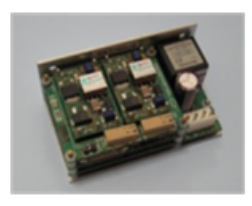

(d)
Figure 1. a) WABIAN-2; b) SABIAN; c) Driver TD12770-48W 10; d) Driver TD12770-48W 05

\section{SABIAN: Sensors, Electronics and Software}

Each joint of SABIAN is equipped with an encoder integrated with the corresponding actuator. These encoders provide 1024 pulses per rotation giving a resolution of $0.35^{\circ}$. The encoders are incremental: so, to give an absolute reference, 16 ON/OFF photo sensors are used. Furthermore, motors provide also a voltage signal proportional to the motor output torque through their Servo Driver modules. The resolution of the provided torque monitoring is equal to $0.4 \%$ of the maximum motor torque. Between each foot and leg of SABIAN there is a six axis force/torque sensor. This sensor can measure a maximum force and torque of $2000 \mathrm{~N}$ and $105 \mathrm{Nm}$, respectively, with an accuracy lower than $1 \%$ of the full scale.

There are also two different versions of the Servo Drivers: TD12770-48W 05 and TD12770-48W 10 (Figure 1c and $1 \mathrm{~d}$ ), able to provide respectively 15 and 30 A to the motors, with a maximum voltage of $48 \mathrm{~V}$. The drivers have the only task to provide the desired outputs and to provide to the motherboard a voltage signal proportional to the motor torque, but without any low-level control. The motherboard PCI-6881 is connected, with a PCI bus, to a JR3 board for 
the reading of the six axis force/torque sensors and with 1 HRP interface board to read the encoder signals, the motor torque, the photo sensors, and to give the 16 analog inputs to the servo drivers. The HRP interface boards are equipped with 2 Digital-to-Analog Converters and 2Analog-to-Digital Converters.

The walking control system of the SABIAN is similar to the WABIAN-2 one and it has two execution phases. The first phase uses a pattern generator that calculates the trajectory of the end-effectors (represented by the feet). In the second phase, the trajectories to be executed are sent to the robot. The SABIAN Pattern Generator uses the feet positions file in order to create the motion pattern of the lower limbs. For generating the correct feet positions, the Pattern Generator needs to know in the best possible way the mass distribution on SABIAN.

At this aim, a new method for the minimization of the errors between a real humanoid robot and a virtual model was studied and implemented[11]. The method allowed reducing the errors in the ZMP motions and in the joint positions, achieving a better repeatability and predictability of the robot behavior.

Additionally, for the dynamic balance of the humanoid robot during the walking stages, legs and waist motions are corrected by a walking stabilization control[13][14] that is based on the ZMP position. In this way we have an ideal motion pattern, and a real one with the online corrections due to the control. Two kind of control are used during the walking motion, the walking stabilization control, that uses the data of the force-torque sensors to stabilize the robot by calculating the ZMP position and the virtual compliance control[15] that reduces the impact of the feet with the ground. Some small modifications were introduced in the code to take care of the different number of load cells ( 2 instead of 4) and to reduce the errors.

\section{SABIAN: Mechanical Optimization}

In the SABIAN structure only 16 actuated joints are actually present. The joints are actuated by three different kinds of DC Maxon motors: 5 DC motors of 22 Watt maximum power and 9 DC motors of 90 Watt maximum power; 2 DC motors of 150 Watt maximum power are used for the knee pitches. A particular role is played by the harmonic drives (HDs), used as speed reducers and torque amplifiers of the DC Maxon motors. The motion is transmitted from the DC motors to the HDs through the belt connecting the pulley fixed to the motor shaft to the one fixed to the HD shaft. Three different kinds of Harmonic Drives are used on the SABIAN structure connected with the three different typologies of motors adopted in SABIAN. The 90 Watt motor responsible for the roll motion of the waist is directly connected to the HD without the belt.

A difference between the WABIAN-2 and SABIAN robots can be here underlined; due to the difference in the Waves Generators of the Harmonic Drives in theWABIAN-2, the HD transmission shaft is directly connected to the Wave Generator and the motion is transmitted with the nut. In the SABIAN the HDs are composed by a cylindrical element with a simple central hole and 4 holes, used to fasten the screws, with the same radial distance from the centre. So, for the SABIAN HDs, an innovative element was developed to incorporate the joint with the shaft, reducing the transmission errors. For the direct connection between the 90 Watt motors of the waist and the HD, a steel hub was designed and implemented. Figure 2 shows the CAD model and the real images of the innovative implemented solutions.

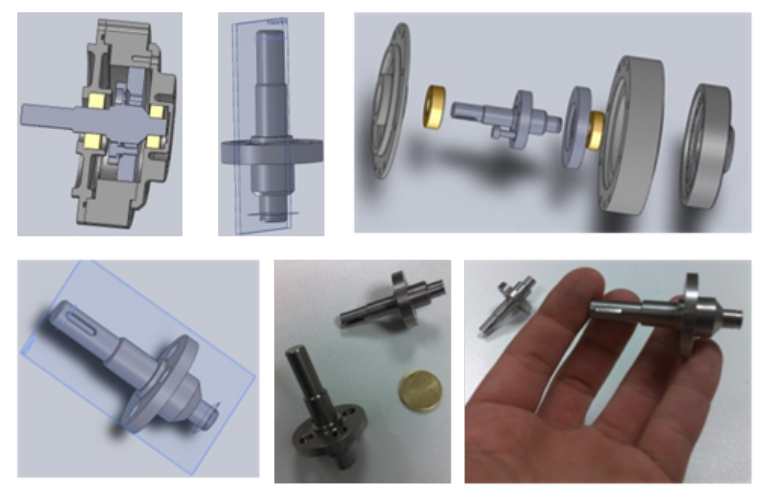

Figure 2. New solution adopted to incorporate the joint with the shaft, reducing the transmission errors. CAD of the new element and images of the effective new solution
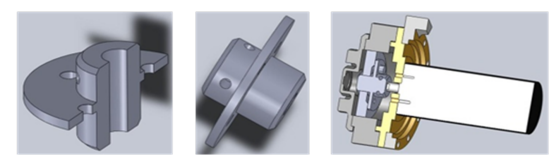

(a)
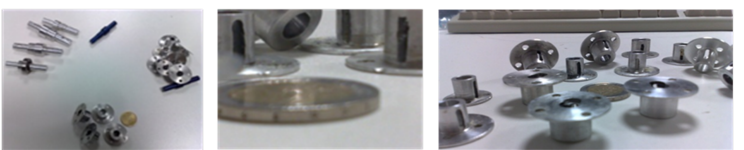

(b)

Figure 3. Previous version of the joints and shafts

With respect to the shafts used in the HDs of theWABIAN-2, the HDs' shafts of the SABIAN are made of a different material. The new solutions are made with steel material in order to reduce the errors related to the damage of the nut on the aluminum shaft. Figure 3 shows the previous version of the shaft with the damage generated by the nut.

At the same time a preliminary study was performed in order to develop and implement an energy harvesting system on the SABIAN platform. Two different gaits (knee-stretched and knee-bended) were performed on the SABIAN with the aim to measure the power consumption of every single joint in different configurations, also in comparison with data related to human movements.

Figure 4 shows a comparison among angle, velocity, joint moment and power of the human knee and SABIAN knee joint during walking at moderate speed. This comparison was carried out after the implementation of optimizations 
presented in this paper on the SABIAN.

Regions of high joint power are best viewed as potential regions for energy harvesting. The swing extension phase is the region in which the largest amount of joint power is dissipated by the knee pitch, while also in stance flexion and in pre-swing phases a huge amount of power is required.

It is remarkable how the energy dissipation trends of human and SABIAN during walking at moderate speed are quite similar. This is due to the human-like movement of the SABIAN joints.

The hip joint of the SABIAN is shown in Figure 5a) and represents, behind the knee, the joint of the robot with the biggest energy consumption during walking. Figure 5a) shows also a sketch of the positioning of alternators for belt tensioning in order to recover energy.
Joint data for the human knee during walking at moderate speed
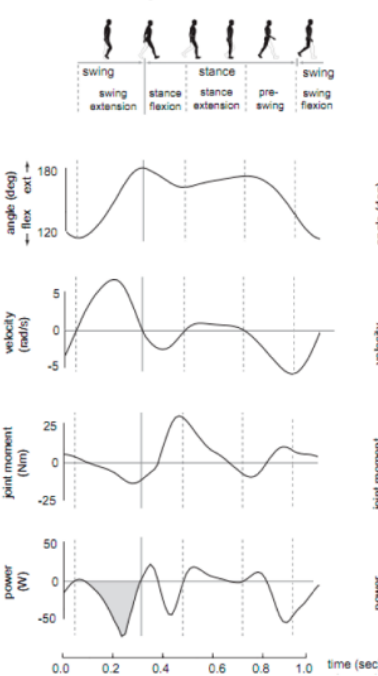

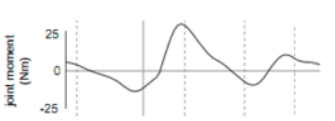

Joint data for Sabian
moderate speed
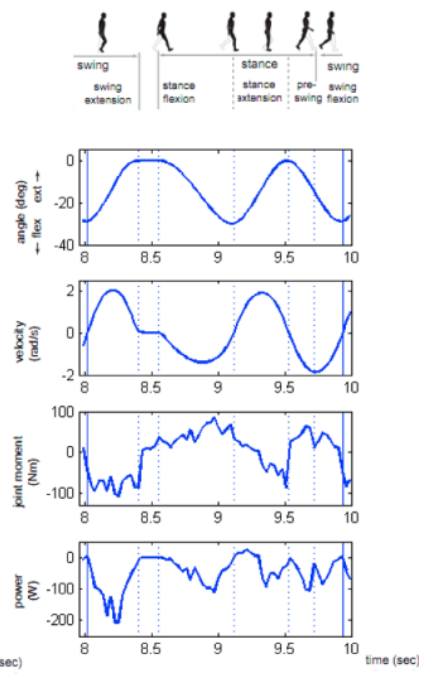

Figure 4. Joint data for the human knee and the SABIAN knee during walking at moderate speed

\section{Conclusions and Future Works}

The humanoid platform SABIAN was presented in this paper with a deep comparison with its Japanese version WABIAN-2. The innovative solutions adopted in the Italian platform, such as the incorporation of the joint with the shaft and a new method for the exact calculation of the error mass[11] are described in details with regards of the previous issues.

With a complete mechanical and electronics review, and the described improvements, SABIAN is now able to walk with a great stability. The robotic platform is currently used in the RoboSoM (A Robotic Sense of Movement, contract No. 248366) European project, which has the objective to investigate new neuroscience-based approaches to the design and development of humanoid robots with advanced perception and action capabilities, showing robust, adaptive and predictive behavior in the real world. The proposed new approaches are strongly based on the concept of human's sense of movement[16].
Many other improvements are planned for the next future: the most important ones are related to the development of a energy harvesting system and to a bio-inspired neck. Regarding the first point, a preliminary work was conducted and presented in the paper.

The analysis of the absorption peaks, especially in the pitch joints of the ankle, the knee and the hip (Figure 4) will give the bases for the designing of a system for recovering energy that will make use of alternators instead of fixed bearings for belt tensioning (Figure $5 \mathrm{a}$ ).

The second planned major modification is related to the integration of the $\mathrm{iCub}[9]$ head on the SABIAN structure (Figure $5 \mathrm{~b}$ ). The subsystems will communicate each other allowing the motion of the robot in an unstructured scenario. From a mechanical viewpoint, a first interface allowing different positions of the head has been developed. Varying the height and the longitudinal position of the head, many tests will be conducted aiming to define the design of a human-like neck.

Other improvements will be done on the SABIAN hardware architecture. In particular, three $25,6 \mathrm{~V}-10 \mathrm{Ah}$ batteries, necessary to keep the robot completely "unwired", with autonomy of 1 hour, are already been evaluated and purchased.

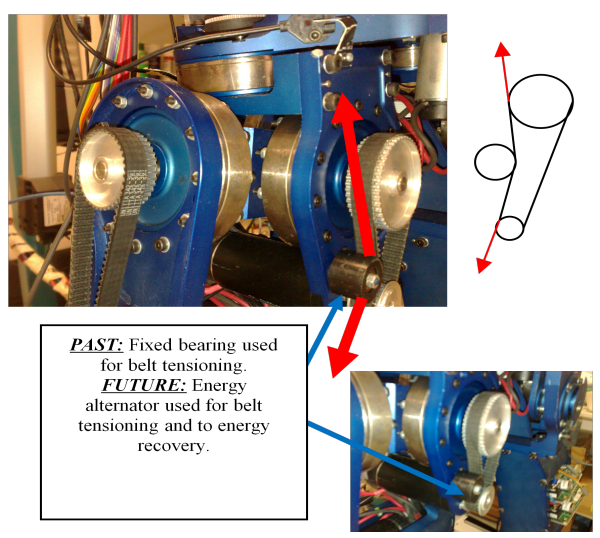

(a)
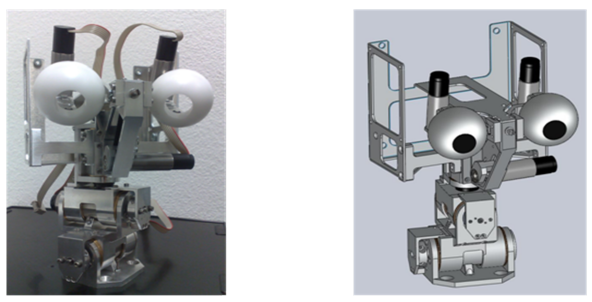

Figure 5. Future works a) Alternators for belt tensioning and energy harvesting b) iCub head currently mounted on SABIAN

Tests on the influence of the opening angle of the feet on the motion stability are also planned. These tests, based on the observation of the human walk, are allowed by the presence of the active jaw joints on the ankle of the robot, a characteristic not present in the current WABIAN-2 structure. Other changes concern the modification of one of the routing boards with a smaller PCB, modifications to enhance the 
compliance of the waist roll motor, a new version of the servo drivers with a low-level control on the torque of the motor, the realization of the SABIAN's compliant arms and the development of bio-inspired feet, with a passive joint for a heel-toe walking and a more anatomically correct shape, are also parts of future steps.

With these other improvements and other modifications not yet planned, the Italian platform SABIAN is going to be a modern humanoid robot able to be used for new challenge in the robotic and neuro-scientific research.

\section{ACKNOWLEDGMENTS}

This research was conducted at the RobotAn Lab, in The BioRobotics Institute of the Scuola Superiore Sant'Anna; and was partly supported by the Italian Ministry of Foreign Affairs, supporting the Robot-An and the RoboCasa joint labs of the Scuola Superiore Sant'Anna and Waseda University and by the European Commission in the ICT STREP RoboSoM Project (contract No. 248366).

\section{REFERENCES}

[1] K. Yokoyama, H. Handa, T. Isozumi, Y. Fukase, K. Kaneko, F. Kanehiro, Y. Kawai, F. Tomita, and H. Hirukawa, "Cooperative works by a human and a humanoid robot", in Proc. ICRA, 2003, pp.2985-2991.

[2] Yu Ogura, Kazushi Shimomura, Hideki Kondo,Akitoshi Morishima, Tatsu Okubo, Shimpei Momoki, Hun-ok Lim, Atsuo Takanishi: Human-like Walking with Knee Stretched, Heel-contact and Toe-off Motion by a Humanoid Robot. IROS 2006

[3] Shinichiro Nakaoka, Atushi Nakazawa, Fumio Kanehiro, Kenji Kaneko, Mitsuharu Morisawa and Katsushi Ikeuchi, "Task Model of Lower Body Motion for a Biped Humanoid Robot to Imitate Human Dances", Proc. 2005 IEEE/RSJ International Conference on Intelligent Robots and Systems, pp.2769-2774, August 2005.

[4] Miomir Vukobratovi' c, "Zero-Moment Point thirty five years of its life", International Journal of Humanoid Robotics Vol. 1, No. 1 (2004) 157-173, World Scientific Publishing Company.

[5] Kajita, S.; Kanehiro, F.; Kaneko, K.; Fujiwara, K.; Harada, K.; Yokoi, K.; Hirukawa, H.; , "Biped walking pattern generation by using preview control of zero-moment point," Proceedings. ICRA '03. IEEE International Conference on Robotics and Automation, 2003, vol.2, pp. 1620-1626, 14-19 Sept. 2003

[6] Ferreira, J.P.; Crisostomo, M.; Coimbra, A.P.; "ZMP trajectory reference for the sagittal plane control of a biped robot based on a human CoP and gait,". IROS 2009. IEEE/RSJ International Conference on Intelligent Robots and Systems,
2009, pp.1588-1593, 10-15 Oct. 2009.

[7] Lim H. O.; Takanishi, A.; , " Locomotion and Compensatory Pattern Generation of Biped Humanoid Robot WABIAN-RV," Journal of the Institute of Image Information and Television Engineers, Vol. 57, No. 1, pp. 63-66, January 2003.

[8] Asfour, T., Regenstein, K., Azad, P., Schröder, J., Vahrenkamp, N. \& Dillmann, R., "ARMAR-III: An Integrated Humanoid Platform for Sensory-Motor Control", In IEEE/RAS International Conference on Humanoid Robots (Humanoids). Genova, Italy. December 2006., pp. 169-175.

[9] Metta, G.; Vernon, D.; Natale, L.; Nori, F.; Sandini, G. "The iCub humanoid robot: an open platform for research in embodied cognition". Workshop on Performance Metrics for Intelligent Systems Workshop. Washington, USA, August 19-21, 2008.

[10] Ogura, Y.; Aikawa, H.; Shimomura, K.; Morishima, A.; Hun-ok Lim; Takanishi, A.; , "Development of a new humanoid robot WABIAN-2," Proceedings ICRA 2006. IEEE International Conference on Robotics and Automation, 2006, pp.76-81, 15-19 May 2006.

[11] Muscolo, G.G.; Recchiuto, C.T.; Laschi, C.; Dario, P.; Hashimoto, K.; Takanishi, A.; , "A method for the calculation of the effective Center of Mass of humanoid robots," 11th IEEE-RAS International Conference on Humanoid Robots (Humanoids 2011), 2011, pp.371-376, 26-28 Oct. 2011, Bled - Slovenia.

[12] Hashimoto, K.; Takezaki, Y.; Hattori, K.; Kondo, H.; Takashima, T.; Lim, H. O.; Takanishi, A., "A Study of Function of the Human's Foot Arch Structure Using Biped Humanoid Robot," Proceedings of the 2010 IEEE/RSJ International Conference on Intelligent Robots and Systems, pp. 2206-2211, October, 2010.

[13] Hun-ok Lim and Atsuo Takanishi (2006). Mechanism and Control of Anthropomorphic Biped Robots, Mobile Robotics, Moving Intelligence, Jonas Buchli (Ed.), ISBN: 3-86611-284-X, InTech.

[14] Kang, H. J.; Momoki, S.; Kondo, H.; Hashimoto, K.; Lim, H. O.; Takanishi, A., "Attitude Compensation Control for Biped Humanoid Robot," Proceeding of the 12th International Conference on Climbing and Walking Robots and the Support Technologies for Mobile Machines (CLAWAR 2009), pp. 677-684, September, 2009.

[15] Sugahara, Y.; Hosobata, T.; Mikuriya, Y.; Lim H. O.; Takanishi, A., "Realization of Stable Dynamic Walking by a Parallel Bipedal Locomotor on Uneven Terrain Using a Virtual Compliance Control," Proceedings of the 2003 IEEE/RSJ International Conference on Intelligent Robots and Systems, pp. 595-600, October, 2003.

[16] A. Berthoz, "The Brain's Sense of Movement". Cambridge, MA/London, 1591, U.K.: Harvard Univ. Press, 2000. 